\title{
Altering Linear Scaling Relationships on Metal Catalysts via Ligand - Adsorbate Hydrogen Bonding
}

\author{
Alexander H. Jenkins, Charles B. Musgrave*, and J. Will Medlin* \\ Department of Chemical and Biological Engineering, University of Colorado Boulder, JSCBB, 3415 Colorado Avenue, \\ Boulder, Colorado 80303, United States
}

\section{Supplementary Information}

Figure S1. DFT functional, unit cell size, and KPOINT mesh comparisons

Figure S2. Bader analysis of thiolate - surface electronic interactions

Figure S3. Steric and geometric effects for thiolate - adsorbate hydrogen bonds

Figure S4. Model competitive reaction system with poisoning from desired product

Figure S5. Energetic penalties for thiolate $\mathrm{C}-\mathrm{C}$ rotation

Figure S6. TGA conformational changes upon forming hydrogen bonds with hydroxymethyl

Figure S7. Adjusting scaling relations to eliminate offsetting geometric and steric effects

Figure S8. Linear scaling relationships for ${ }^{*} \mathrm{O}$ and ${ }^{*} \mathrm{OH}$ vs ${ }^{*} \mathrm{OO}$ on thiolate-modified surfaces

Figure S9. Estimated $\mathrm{H}$-bond strength vs $\mathrm{O}-\mathrm{H}$ bond lengthening for ${ }^{*} \mathrm{O}$

Figure S10. *OO interacting with various TGA-modified surfaces

Table S1. Calculated Adsorption Energies for Linear Scaling Relationships

Method S1. Model competitive reaction system 

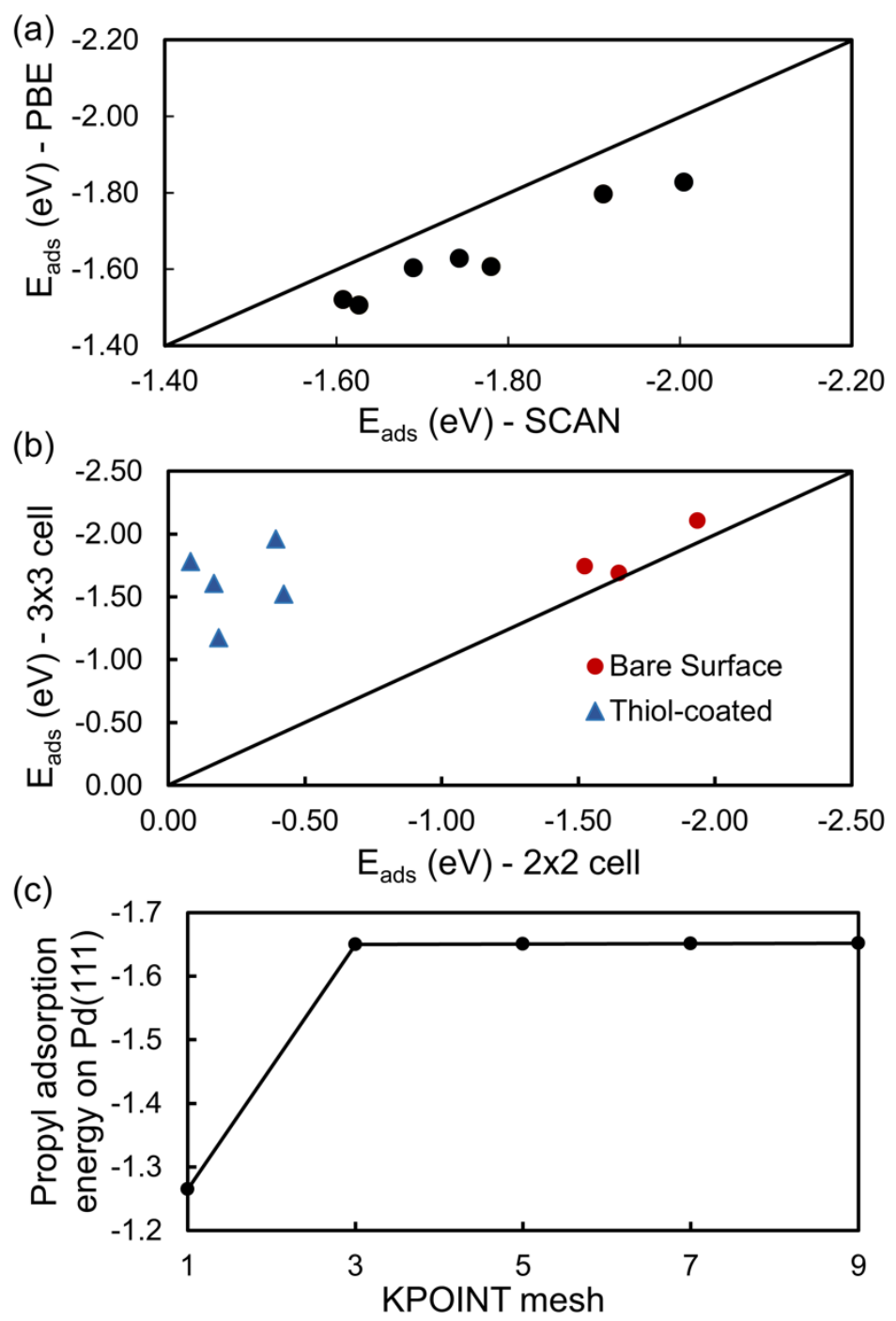

Figure S1. (a) Parity plot comparing alkyl and hydroxyalkyl adsorbate binding on Pd with PBE and SCAN functionals. (b) parity plot comparing alkyl and hydroxyalkyl adsorbate binding on $3 \times 3$ and $2 \times 2$ Pd unit cells with and without thiolate-modifiers. (c) Propyl adsorption energy on $\mathrm{Pd}(111)$ vs KPOINT mesh size, showing $3 \times 3 \times 1$ KPOINT mesh is sufficient. 

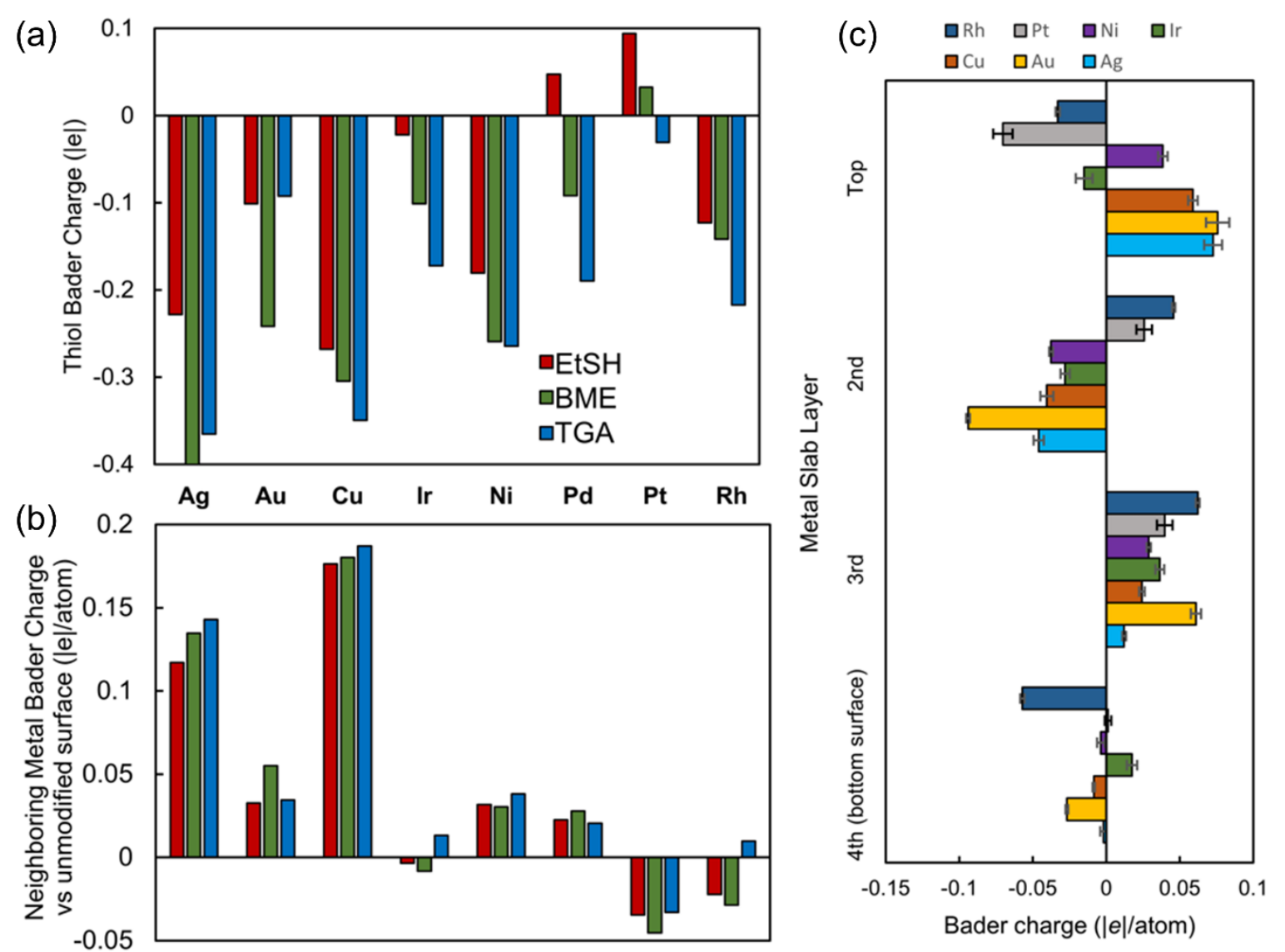

Figure S2. (a) Total Bader charge of thiolates adsorbed to fcc (111) surface. (b) Average change in Bader charge of the four nearest surface metal atoms following thiolate adsorption. (c) The average Bader charge of each layer of the metal slab, following thiolate adsorption. Error bars show the standard deviation between calculations with EtSH-, BME-, and TGAmodified surfaces. 


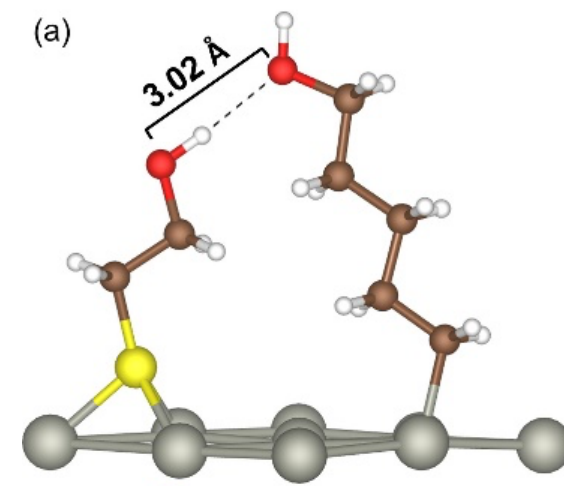

(b)

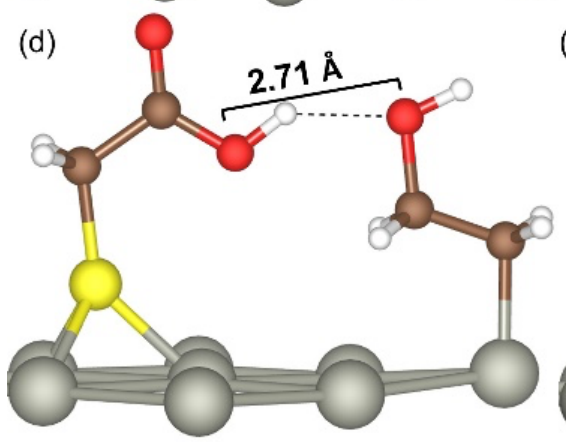

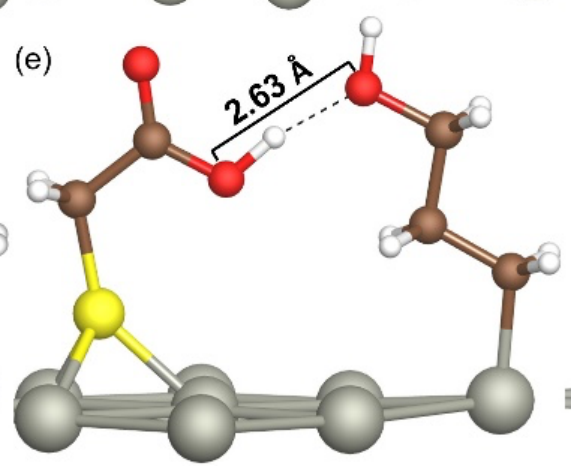

(c)

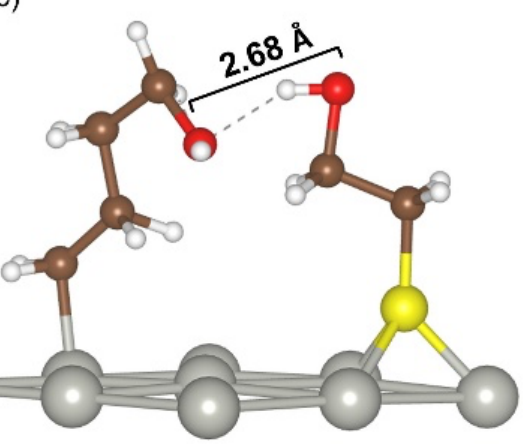

(f)

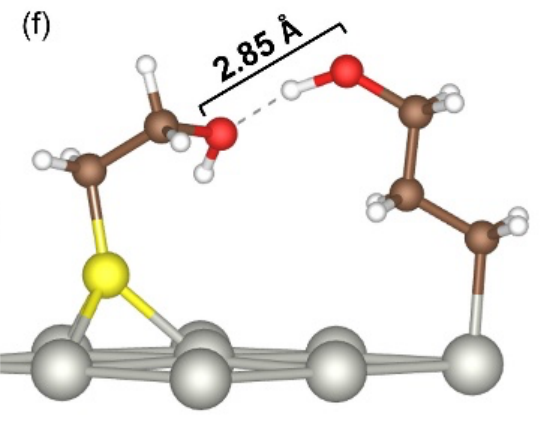

Figure S3. Measurements shown describe the distance between $\mathrm{O}$ atoms for the $\mathrm{H}$-bond acceptor and donor groups. (a) The O-O distance between BME and the hydroxypentyl is short enough for $\mathrm{H}$-bonding to be possible. (b) Steric hindrance keeps $\mathrm{O}$ atoms further apart for hydroxybutyl in this orientation, hindering $\mathrm{H}$-bonding. (c) Rotating the $\mathrm{C}-\mathrm{C}$ bond of hydroxybutyl removes steric hindrance and allows for $\mathrm{H}$-bonding (d) $\mathrm{H}$-bonding interactions between TGA and hydroxyethyl are $0.16 \mathrm{eV}$ weaker than with (e) hydroxypropyl when the distance between adsorption sites is large due to a longer $\mathrm{O}-\mathrm{O}$ distance. (f) BME can also act as an $\mathrm{H}$-bond acceptor. 

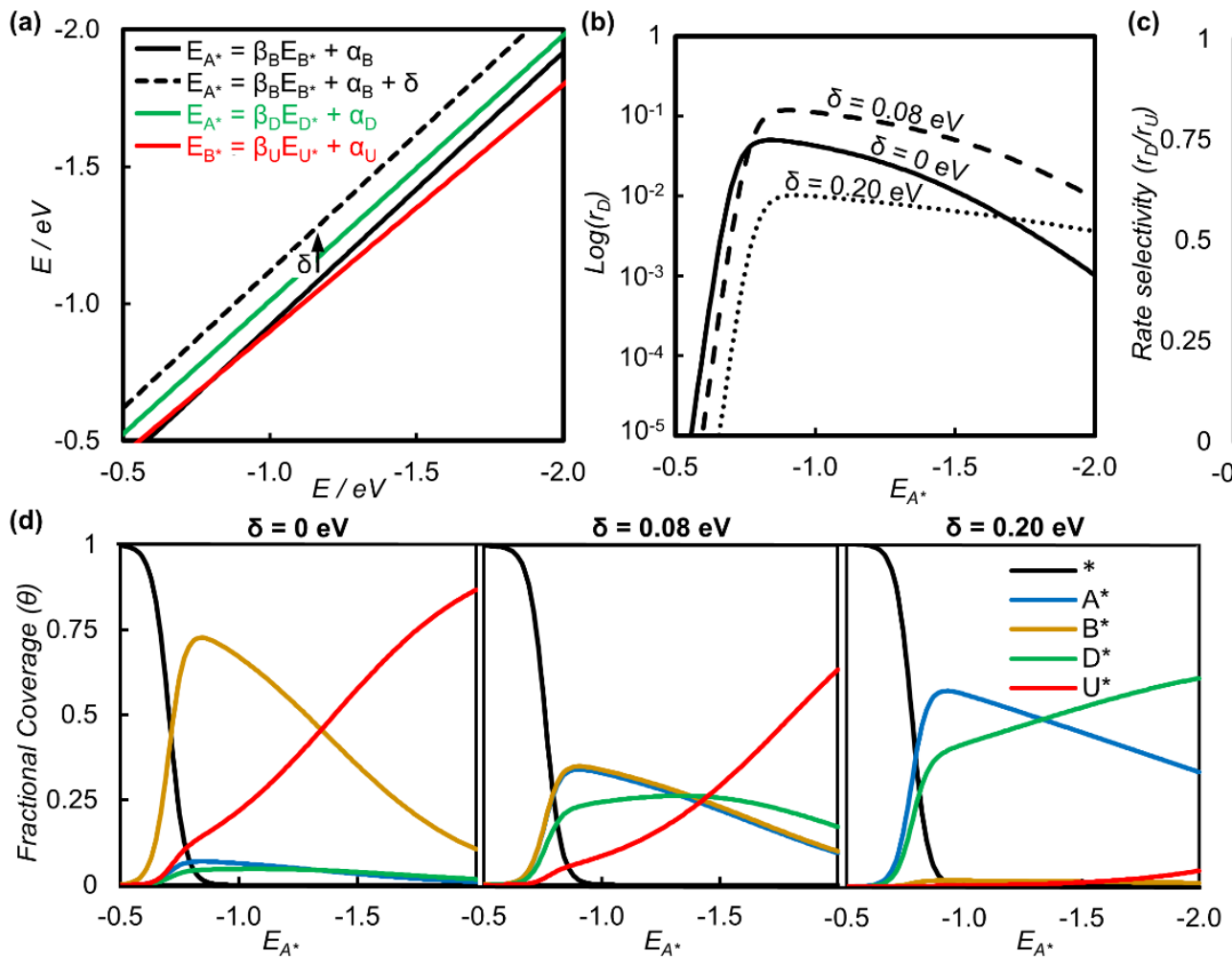

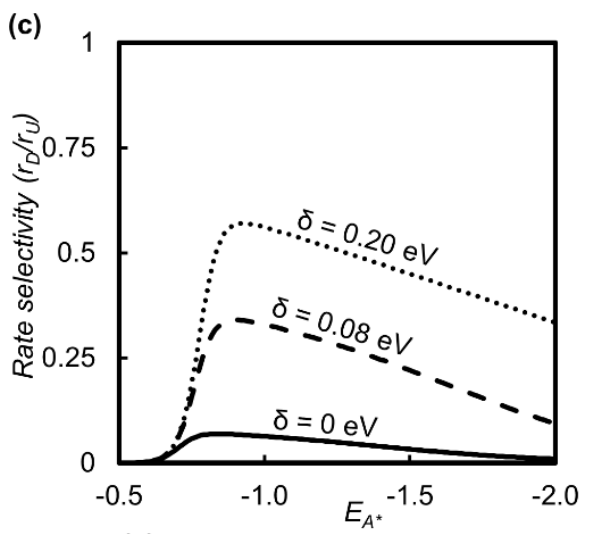

(e)

Reaction Network:

(1) $A_{(g)} \rightleftharpoons A^{*}$

(2) $\mathrm{B}_{(\mathrm{g})} \rightleftharpoons \mathrm{B}^{*} \rightarrow \mathrm{U}^{*} \rightleftharpoons \mathrm{U}_{(\mathrm{g})}$

(3) $A^{*}+B^{*} \rightarrow D^{*} \rightleftharpoons D_{(g)}$

$r_{D} \propto \theta_{A^{*}} \theta_{B^{*}}$

$r_{U} \propto \theta_{\mathrm{B}^{*}}$

$H$-bond forming: $A^{*} \& D^{*}$ Non-H-bond forming: $\mathrm{B}^{\star} \& \mathrm{U}^{*}$

$p_{A}=p_{B}=p_{D}=100 p_{U}$

Figure S4. Additional model system, identical to that of Figure 3 except: $p_{D}=p_{A}=p_{B}=100 p_{U}$. This results in site poisoning from $D^{*}$, especially for high $\delta$. (a) Linear scaling relationships for $A^{*}$ vs $D^{*}$ (green), $B^{*}$ vs $U^{*}$ (red), $A^{*}$ vs $B^{*}$ (solid black), and $A^{*}$ vs $B^{*}$ following a shift in y-intercept of magnitude $\delta$ (dashed black). (b) Volcano-like rate dependence of the desired reaction pathway on $E A^{*}$ for different magnitude shifts $(\delta)$. A shift of $\delta=0.08 \mathrm{eV}$ represents a catalyst optimized to maximize $r_{D}$. (c) Rate selectivity towards the desired reaction pathway. (d) Calculated fractional surface coverages of each adsorbate for varying magnitudes of $\delta$. (e) Reaction model explanation. Additional information on the methods and parameters used for this model can be found in Method S1 in the Supporting Information. 
(a)

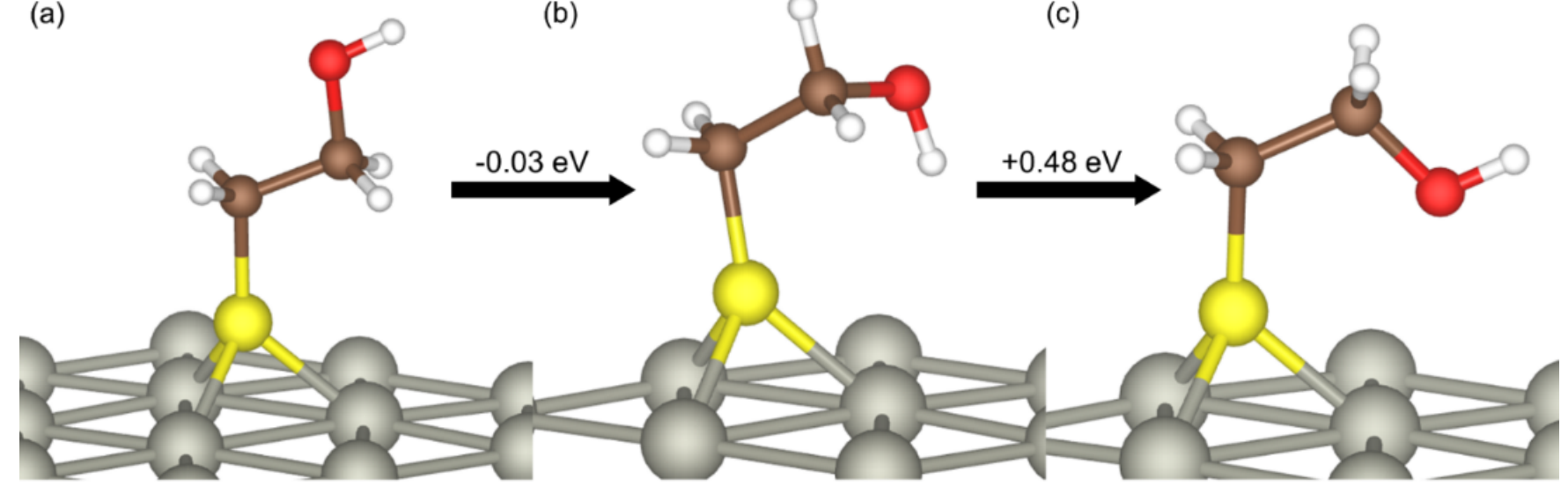

Figure S5. BME-modified $\mathrm{Pd}$ (111) surfaces in different orientations. The energy penalty for $120^{\circ}$ rotation along the $\mathrm{C}-\mathrm{C}$ bond is $0.03 \mathrm{eV}(\mathrm{a}-\mathrm{b})$, whereas the penalty for the overlapping configuration (c) is $0.48 \mathrm{eV}$ 
(a)

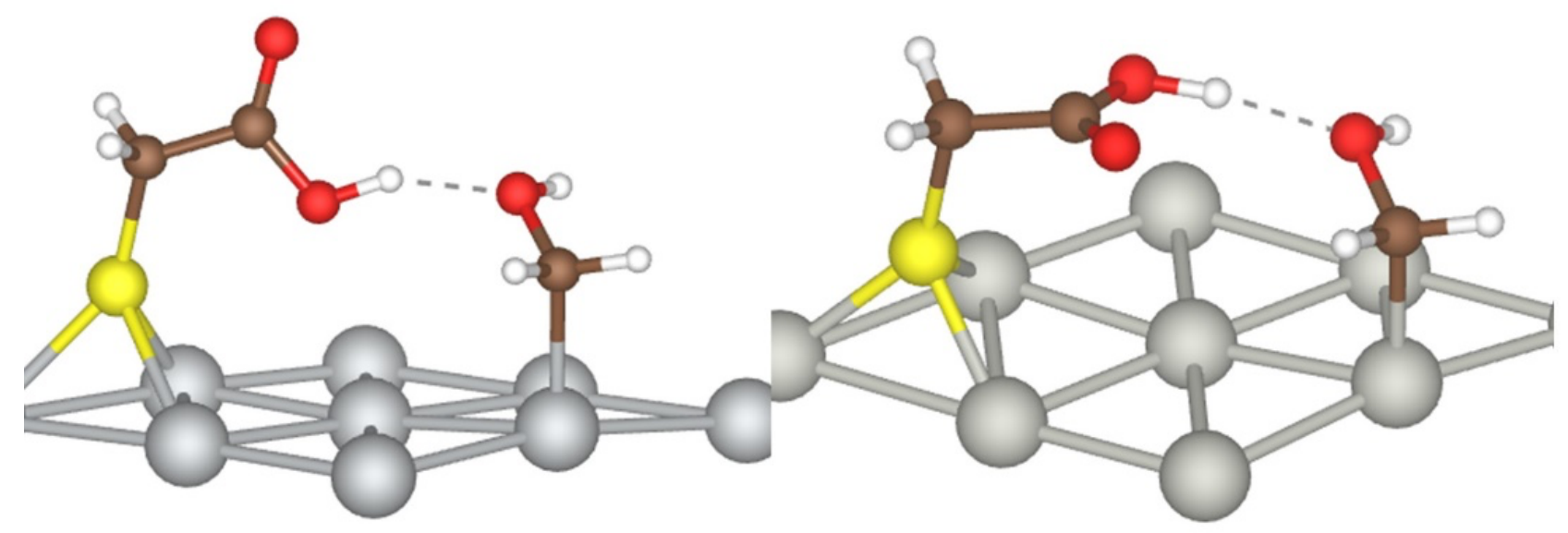

Figure S6. TGA/MeOH on $\mathrm{Ag}(\mathrm{a})$ and $\mathrm{Pd}$ (b). TGA undergoes a conformational change on strongly-binding metals such as $\mathrm{Pd}$ in order to facilitate $\mathrm{H}$-bonding 


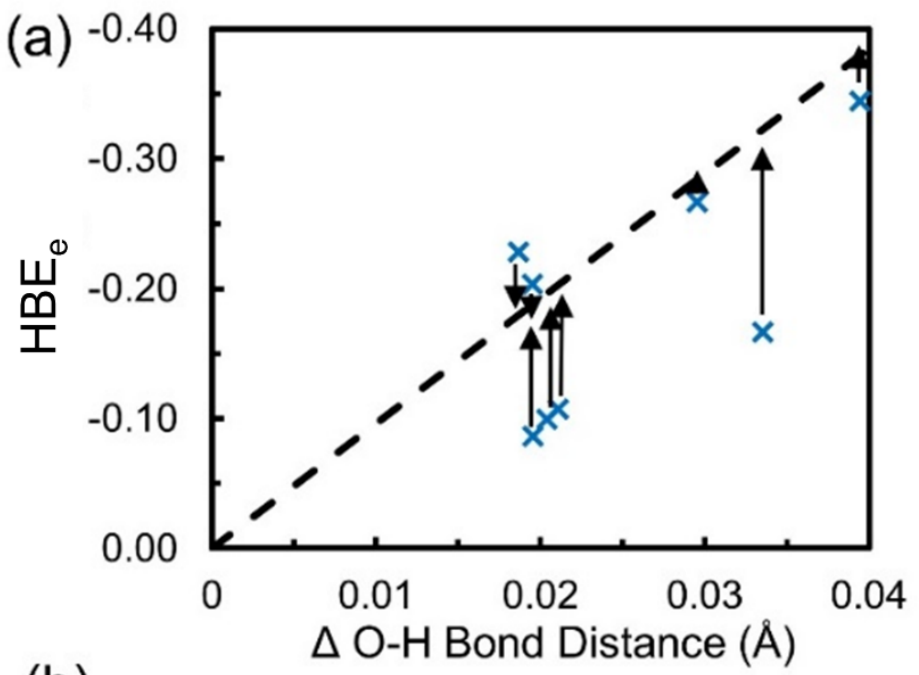

(b)
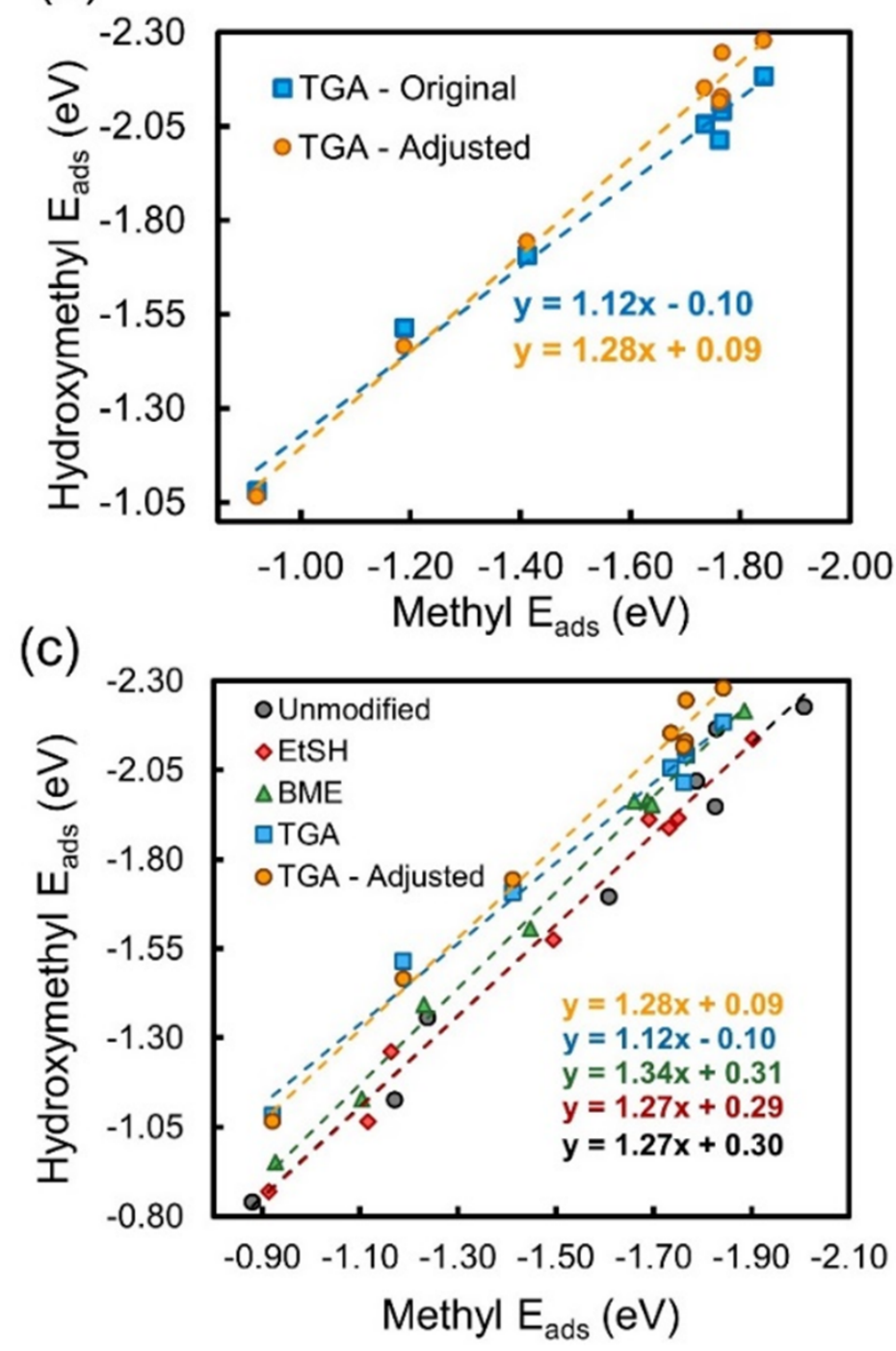

Figure S7. (a) $\mathrm{O}-\mathrm{H}$ length increases with estimated $\mathrm{H}$-bond strength due to delocalization of the proton. Blue crosses show O-H bond lengthening for TGA with hydroxymethyl on the surface. Arrows represent manual shift of binding strength based on predictions from $\mathrm{O}-\mathrm{H}$ bond lengthening. (b) Adjusted scaling of methyl vs hydroxymethyl based on shifted binding strengths compared to original scaling curve. (c) Adjusted TGA scaling curve has similar slope to unmodified and EtSH-coated surfaces. 

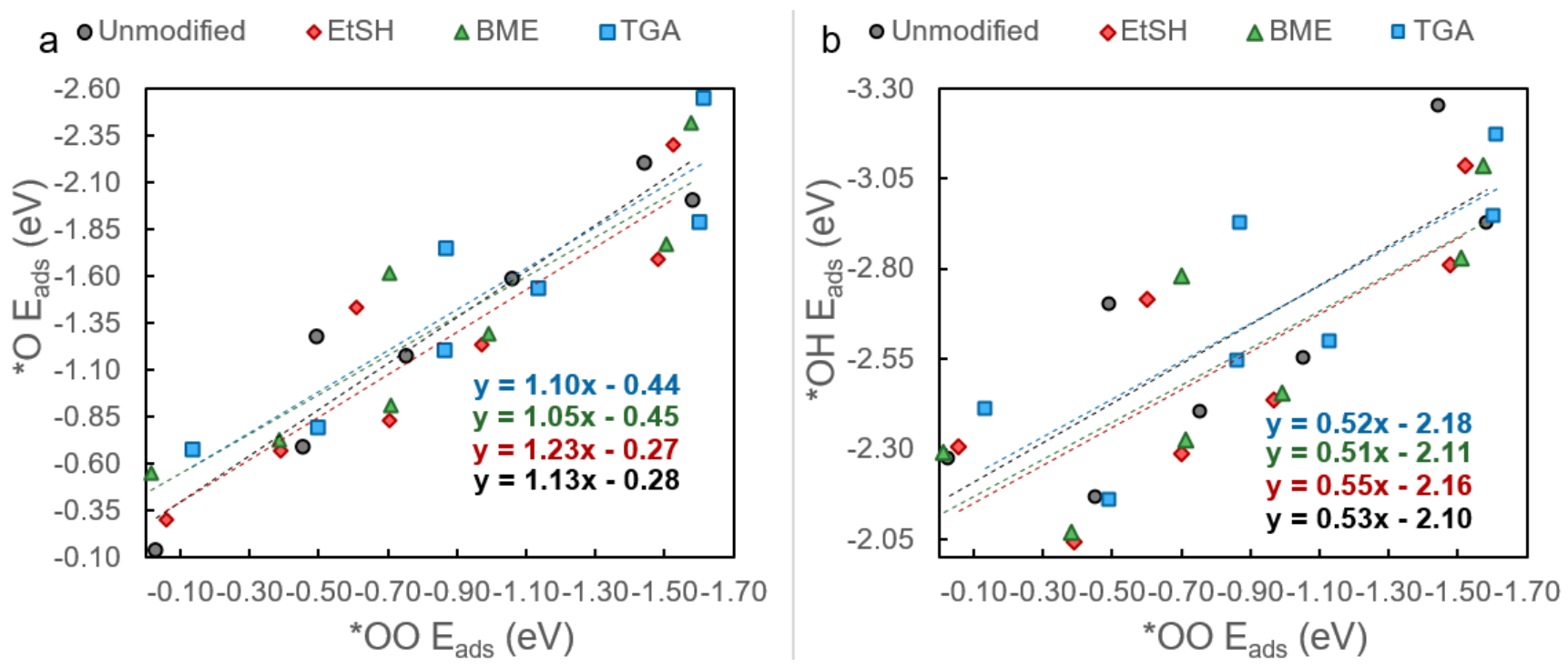

Figure S8. (a) Adsorption energies of ${ }^{*} \mathrm{O}$ vs ${ }^{*} \mathrm{OO}$ on thiolate-modified fcc (111) surfaces. (b) Adsorption energies of ${ }^{*} \mathrm{OH}$ vs ${ }^{*} \mathrm{OO}$ on thiolate-modified fcc (111) surfaces. Both scaling graphs show significant scatter compared to hydroxyalkyl systems due to O-M interactions 


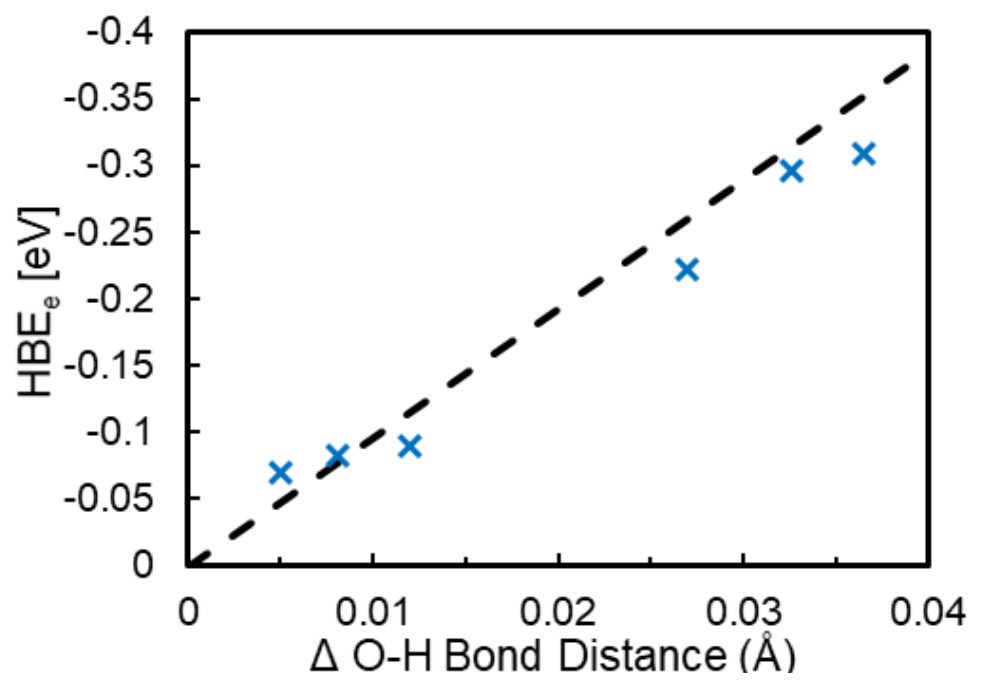

Figure S9. The $\mathrm{y}$-axis shows estimated $\mathrm{H}$-bond strength for systems with ${ }^{*} \mathrm{O}, \mathrm{HBE}_{\mathrm{e}}$, where ${ }^{*} \mathrm{OO}$ acts as the 'alkyl' adsorbate in Eqn(1). The dashed line shows predicted $\mathrm{H}$-bond strength based on $\triangle \mathrm{O}-\mathrm{H}, \mathrm{HBE}_{\mathrm{g}}$, as established in Figure 4 and $\mathrm{Eqn}(2)$. On average, $\mathrm{HBE}_{\mathrm{e}}$ is only .02 eV lower than $\mathrm{HBE}_{\mathrm{g}}$, indicating minimal non-H-bonding effects. 

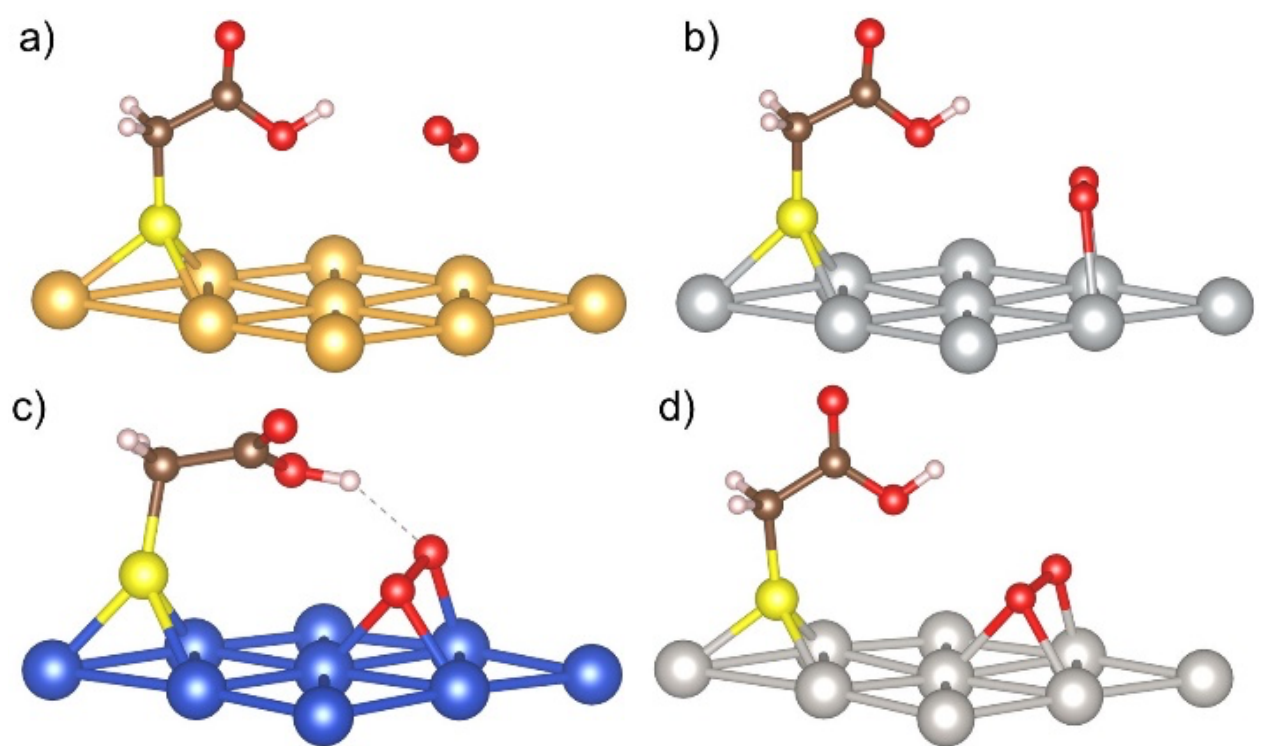

Figure S10. *OO adsorption on TGA-modified a) Au, b) $\mathrm{Ag}, \mathrm{c}$ ) $\mathrm{Cu}$, and d) Pt. All metals not shown resembled the Pt system. 
Table S1. Calculated Adsorption Energies for Linear Scaling Relationships [eV]

\begin{tabular}{|c|c|c|c|c|c|c|c|}
\hline Surface & $M e$ & $\mathrm{MeOH}$ & $P r$ & $\mathrm{PrOH}$ & 0 & $\mathrm{OH}$ & 00 \\
\hline$A g(111)$ & -0.88 & -0.84 & -0.65 & -0.68 & -0.14 & -2.27 & -0.03 \\
\hline$A g(111)-E t S H$ & -0.91 & -0.87 & -0.69 & -0.74 & -0.30 & -2.31 & -0.06 \\
\hline$A g(111)-B M E$ & -0.93 & -0.95 & -0.74 & -0.89 & -0.55 & -2.29 & -0.01 \\
\hline$A g(111)-T G A$ & -0.92 & -1.08 & -0.66 & -1.06 & -0.68 & -2.41 & -0.13 \\
\hline$A u(111)$ & -1.24 & -1.36 & -1.06 & -1.06 & +0.44 & -1.72 & +0.00 \\
\hline$A u(111)-E t S H$ & -1.16 & -1.26 & -0.97 & -1.01 & +0.39 & -1.77 & -0.06 \\
\hline$A u(111)-B M E$ & -1.23 & -1.39 & -1.03 & -1.18 & +0.22 & -1.74 & +0.01 \\
\hline$A u(111)-T G A$ & -1.19 & -1.51 & -0.87 & -1.23 & -0.27 & -1.92 & +0.20 \\
\hline $\mathrm{Cu}(111)$ & -1.17 & -1.12 & -0.93 & -0.96 & -1.28 & -2.70 & -0.49 \\
\hline$C u(111)-E t S H$ & -1.12 & -1.07 & -0.88 & -0.92 & -1.43 & -2.72 & -0.60 \\
\hline$C u(111)-B M E$ & -1.10 & -1.13 & -0.86 & -1.08 & -1.62 & -2.78 & -0.70 \\
\hline$C u(111)-T G A$ & -1.41 & -1.71 & -0.88 & -1.31 & -1.75 & -2.92 & -0.87 \\
\hline $\operatorname{Ir}(111)$ & -1.83 & -1.95 & -1.67 & -1.69 & -1.59 & -2.55 & -1.06 \\
\hline $\operatorname{Ir}(111)-E t S H$ & -1.73 & -1.89 & -1.54 & -1.60 & -1.24 & -2.44 & -0.97 \\
\hline $\operatorname{Ir}(111)-B M E$ & -1.69 & -1.96 & -1.55 & -1.75 & -1.29 & -2.46 & -0.99 \\
\hline $\operatorname{Ir}(111)-T G A$ & -1.77 & -2.09 & -1.38 & -1.86 & -1.54 & -2.60 & -1.14 \\
\hline $\mathrm{Ni}(111)$ & -1.61 & -1.69 & -1.41 & -1.44 & -2.20 & -3.25 & -1.44 \\
\hline$N i(111)-E t S H$ & -1.50 & -1.57 & -1.24 & -1.29 & -2.30 & -3.09 & -1.52 \\
\hline$N i(111)-B M E$ & -1.45 & -1.61 & -1.19 & -1.42 & -2.42 & -3.09 & -1.57 \\
\hline$N i(111)-T G A$ & -1.76 & -2.11 & -1.17 & -1.61 & -2.55 & -3.17 & -1.62 \\
\hline$P d(111)$ & -1.79 & -2.02 & -1.65 & -1.67 & -1.17 & -2.40 & -0.76 \\
\hline$P d(111)-E t S H$ & -1.69 & -1.91 & -1.53 & -1.57 & -0.83 & -2.29 & -0.70 \\
\hline$P d(111)-B M E$ & -1.66 & -1.96 & -1.55 & -1.72 & -0.92 & -2.33 & -0.71 \\
\hline$P d(111)-T G A$ & -1.73 & -2.06 & -1.54 & -1.98 & -1.21 & -2.54 & -0.86 \\
\hline $\operatorname{Pt}(111)$ & -2.01 & -2.23 & -1.88 & -1.88 & -0.69 & -2.17 & -0.46 \\
\hline$P t(111)-E t S H$ & -1.90 & -2.14 & -1.74 & -1.78 & -0.66 & -2.05 & -0.39 \\
\hline$P t(111)-B M E$ & -1.89 & -2.22 & -1.77 & -1.96 & -0.72 & -2.07 & -0.38 \\
\hline$P t(111)-T G A$ & -1.84 & -2.18 & -1.73 & -2.17 & -0.79 & -2.15 & -0.50 \\
\hline$R h(111)$ & -1.83 & -2.16 & -1.69 & -1.70 & -2.01 & -2.92 & -1.58 \\
\hline$R h(111)-E t S H$ & -1.75 & -1.92 & -1.57 & -1.61 & -1.69 & -2.81 & -1.48 \\
\hline$R h(111)-B M E$ & -1.70 & -1.95 & -1.56 & -1.76 & -1.77 & -2.83 & -1.51 \\
\hline$R h(111)-T G A$ & -1.76 & -2.01 & -1.59 & -1.98 & -1.90 & -2.95 & -1.60 \\
\hline
\end{tabular}




\section{Method S1.}

The model reaction network in Figure 3 adheres to the following linear scaling relationships (LSRs):

$$
\begin{aligned}
& \mathrm{E}_{\mathrm{A}^{*}}=\beta_{\mathrm{B}} \mathrm{E}_{\mathrm{B}^{*}}+\alpha_{\mathrm{B}} \\
& \mathrm{E}_{\mathrm{A}^{*} \text {,shifted }}=\beta_{\mathrm{B}} \mathrm{E}_{\mathrm{B}^{*}}+\alpha_{\mathrm{B}}+\delta \\
& \mathrm{E}_{\mathrm{A}^{*}}=\beta_{\mathrm{D}} \mathrm{E}_{\mathrm{D}^{*}}+\alpha_{\mathrm{D}} \\
& \mathrm{E}_{\mathrm{B}^{*}}=\beta_{\mathrm{U}} \mathrm{E}_{\mathrm{U}^{*}}+\alpha_{\mathrm{U}}
\end{aligned}
$$

where $\beta_{B}=0.999 ; \beta_{D}=0.970 ; \beta_{U}=0.900 ; \alpha_{B}=0.08 ; \alpha_{D}=-0.04 ; \alpha_{U}=0.00$. These values indicate a well-scaling system, where slight differences in $\alpha$ can be attributed to differing adsorption sites and differences in $\beta$ can be related to the relative coordination of surface-bound groups. ${ }^{1} \delta$, the shift in LSR caused by ligand - adsorbate bonding, can be varied to model different strengths of bonding. These LSRs were used to determine adsorption energies for $B^{*}, D^{*}$, and $U^{*}$ based off of those from $A^{*}$, our independent variable. Values for $E_{A^{*}}$ ranged from $-0.50--2.00 \mathrm{eV}$ and represent metal surfaces of varying reactivities.

Thus, for each given value of $E_{A^{*}},-\Delta H_{a d s}$ was known for each adsorbate. Then the adsorption equilibrium constant, $b$, for each molecule was calculated using the proportionality relationship: $b \propto \mathrm{e}^{-\Delta \mathrm{H}_{a d s} / R T}$, where $T=400 \mathrm{~K}$. A proportionality constant of $1 \times 10^{-10}$ was chosen for each species. The use of this proportionality relationship requires the assumption that enthalpy of adsorption is independent of coverage. Fractional surface coverages were calculated using a competitive Langmuir adsorption model:

$\theta_{A}=\frac{b_{A} p_{A}}{1+b_{A} p_{A}+b_{B} p_{B}+b_{D} p_{D}+b_{U} p_{U}}$,

where $p_{A}=p_{B}=100 \times p_{D}=100 \times p_{U}$. These pressures could exist for a reaction system with low conversion, leading to low pressures of either product. Figure $S 4$ demonstrates a system with higher partial pressures of $D$, leading to poisoning with $D^{*}$.

Finally, these surface coverages were used to calculate rates for $\left(B^{*} \rightarrow U^{*}\right)$ and $\left(A^{*}+B^{*} \rightarrow D^{*}\right)$ based on these relationships, where pre-exponential factors equal unity:

$r_{D} \propto \theta_{A^{*}} \theta_{B^{*}}$

$r_{U} \propto \theta_{B^{*}}$

We calculate selectivity as the ratio of $r_{D}: r_{u}$. An optimal value of $\delta$-that which maximized $r_{D}-$ was calculated with a GRG nonlinear scaling method, varying $\delta$ to maximize $r_{D}$. This optimized $\delta$ is dependent on model parameters. 


\section{Supplementary References}

(1) Montemore, M. M.; Medlin, J. W. Scaling Relations between Adsorption Energies for Computational Screening and Design of Catalysts. Catal. Sci. Technol. 2014, 4 (11), 3748-3761. https://doi.org/10.1039/c4cy00335g. 